\title{
Bladder repair following iatrogenic cystotomy in irradiated small capacity bladders
}

\author{
Jia Yi $\underline{\text { Chee }}^{1}$, MBBs, Pradeep $\underline{\text { Durai }}{ }^{2}$, MBBs, Mei Wen Fiona $\underline{W u^{2}}$, MBBs, Ho Yee Tiong $^{2}$, MD
}

\begin{abstract}
During laparotomy in a previously irradiated and operated pelvis, incidental cystotomies can occur and a tension-free, watertight, two- or three-layer closure of the bladder may be impossible. We herein report two cases of iatrogenic defects of the bladders in post-irradiated pelvises and compare the two different methods of bladder repair employed - an ileal augmentation segment used in the first case and bovine pericardial graft used in the second. Successful closures of the bladder defects were achieved in both cases. Native irradiated bowel and bovine pericardium can be useful substitutes in situations involving bladder defects in a previously irradiated pelvis. The advantages and disadvantages of the two approaches are also herein discussed.
\end{abstract}

Keywords: bladder, bovine pericardium, ileum

\section{INTRODUCTION}

For many decades, the search for an ideal bladder wall substitute has been with limited success. It has been difficult to replace what nature has intended the bladder to be - a compliant, low pressure reservoir for urine, lined by urothelium and controlled by synchronised bladder contractions made possible by detrusor muscle. Bladder wall substitutes are used mainly for augmentation or as partial replacements for diseased or irradiated bladders. ${ }^{(1)}$ During a laparotomy in a previously irradiated and operated pelvis, incidental cystotomies can occur during adhesiolysis or tumour resection performed to obtain clear margins. The traditional method, a tension-free, watertight, two- or three-layer closure, may not be possible due to the unhealthy, small capacity, contracted bladder; such conventional techniques involve a high risk of failure and subsequent fistula formation. We herein report two cases in which we performed difficult cystotomy repairs using different bladder wall substitutes, successfully closing the bladder defects in both cases.

\section{CASE REPORTS}

\section{Case 1}

A 71-year-old woman was treated for cervical cancer via total abdominal hysterectomy and adjuvant chemoradiation 14 years ago. Although she had no recurrent disease post treatment, she suffered from repeated intestinal obstruction due to adhesions and radiation-related rectovesical fistulae. As a result, she had undergone three laparotomies, including a total colectomy. She also had peripheral vascular disease, which was treated via femoral-femoral crossover grafting five years earlier.

The patient subsequently presented at our hospital with another episode of acute intestinal obstruction, which required exploratory laparotomy. During the surgery, dense intraperitoneal adhesions with dense pelvic fibrosis of the small bowel to the pelvis and bladder were found. During adhesiolysis, there was an inadvertent entry into the bladder and a 3-cm defect was sustained at the bladder dome. Following complete mobilisation of the small bowel out of the pelvis, a decision was made to manage her condition with an end ileostomy. After resection of the unhealthy distal bowel, the remnant small bowel measured $140 \mathrm{~cm}$ from the duodenojejunal flexure. The hospital's urologists were consulted and found the bladder to be contracted (to an estimated volume of $50 \mathrm{cc}$ ) and thin-walled, with no significant detrusor layer. There were also extensive adhesions from the bladder to the pelvic sidewall. Retropubic mobilisation for primary closure of the bladder defect was impossible due to the femoral-femoral crossover graft at the suprapubic region. A decision was thus made to use the distal segment of the terminal small bowel (approximately $5 \mathrm{~cm}$ in length) and its mesentery for closure.

The edges of the bladder were freshened, and the $5-\mathrm{cm}$ pedicled terminal ileum was detubularised and opened along its antimesenteric border. The anterior aspect of the enterocystoplasty was closed using continuous Vicryl 2-0 suture, and interrupted PDS II 4-0 suture, which is longer-lasting, was used for closure of the posterior aspect of the enterocystoplasty. Care was taken intraoperatively to ensure that there was, as best as possible, no tension to the closure in order to prevent dehiscence and leakage. A retropubic drain was placed and the urethral catheter was inflated with $5 \mathrm{~mL} \mathrm{H}_{2} \mathrm{O}$. The surgery was subsequently handed back to the colorectal team for completion and closure. The overall operative time was 4 hours, in which the bladder closure took approximately 1 hour.

The patient's initial cystography, performed on Day 10 post operation, showed urinary leakage (Fig. 1). This was treated conservatively via urinary drainage using a Foley catheter and an abdominal Jackson-Pratt drain. On follow-up six weeks post surgery, contrast-enhanced computed tomography (CT) of the abdomen and pelvis showed a healed enterocystoplasty, and the patient was able to void on her own, with an increased bladder capacity (an estimated volume of 80-100 cc) based on frequency 


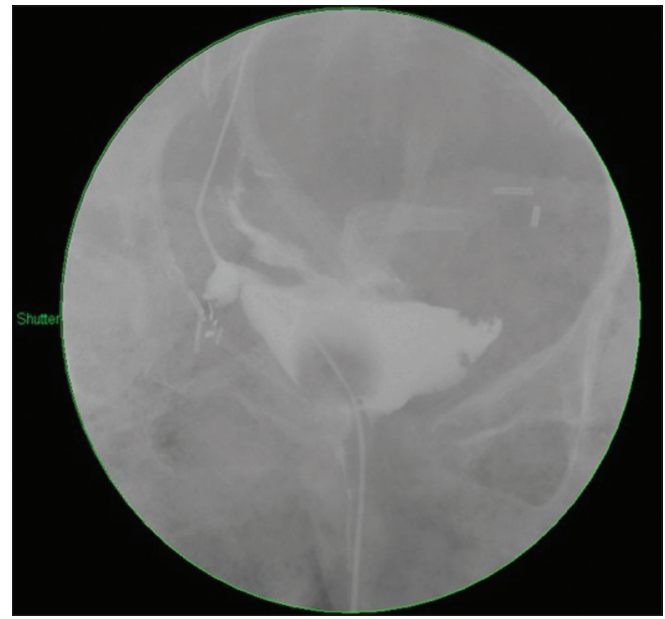

Fig. 1 Cystogram shows leaking anastomosis of the ileal graft.

volume charts. Renal function was maintained at baseline (serum creatinine $[\mathrm{Cr}] 41 \mathrm{mg} / \mathrm{dL}$, estimated glomerular filtration rate [eGFR] $>60 \mathrm{~mL} / \mathrm{min}$ ) at seven months post surgery. Repeat CT showed no leakage at the enterocystoplasty site (Fig. 2), as well as partial resolution of a previous hydronephrosis. Our patient also reported satisfactory voiding function.

\section{Case 2}

A 47-year-old man who had recurrent ascending colon adenocarcinoma following an anterior resection underwent partial cystectomy with a left-to-right uretero-ureteral anastomosis, as well as chemoradiation for rectal cancer 16 years ago. He subsequently presented with a recurrent ascending colon tumour and underwent total colectomy and end ileostomy in the previous year.

Intraoperatively, he was found to have dense adhesions involving the small and remaining large bowels, abdominal wall, retroperitoneum, pelvis, presacral fascia and bladder. A loop of ileum, about $110 \mathrm{~cm}$ proximal to the ileocaecal valve, was found to be densely adherent to the pelvic floor and the posterior part of the bladder. During the separation of these structures, there was an inadvertent injury to the bladder. The hospital's urologists were informed and a decision was made to attempt closure of the bladder defect with bovine pericardium flap. The bowels were unable to be used, as they were extremely unhealthy due to previous extensive radiation, and significantly adherent to the surrounding structures. The bladder, which was thin-walled and small in capacity, was adherent to the pubic bone anteriorly and the pelvic wall laterally. The cystotomy edges were trimmed to the presumed healthy area of the bowel. Primary closure of the bladder defect was made difficult due to previous near-total partial cystectomy and pelvic irradiation. It was also not possible to mobilise the bladder to allow for a tension-free closure. Bovine pericardium patch measuring 10 $\mathrm{cm} \times 15 \mathrm{~cm}$ (Edwards Lifesciences, Irvine, CA, USA) was used as a partial bladder wall substitute. The patch, which measured $4 \mathrm{~cm} \times 4 \mathrm{~cm}$ in our case, was used to close the bladder defect in a cystoplasty manner, and interrupted PDS 3-0 sutures were used for closure. The operation was subsequently handed back

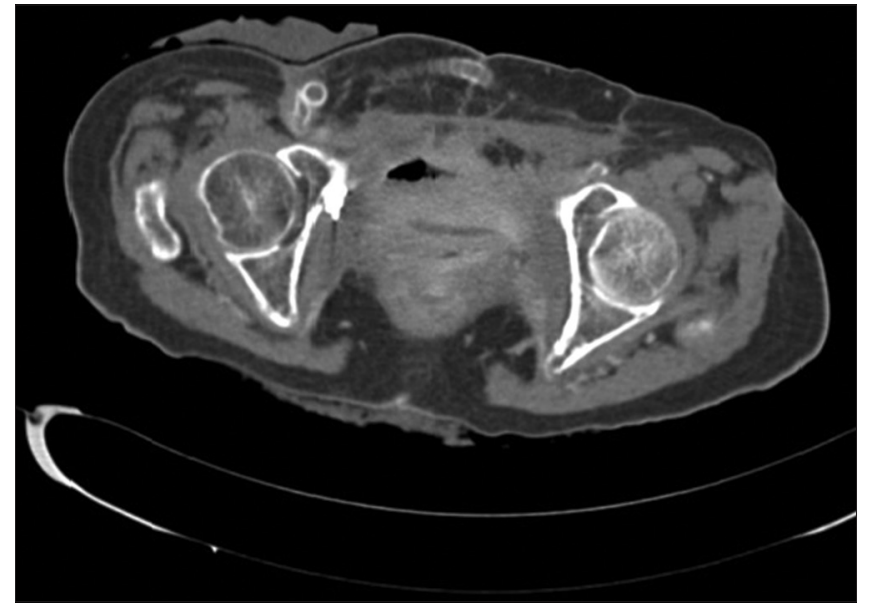

Fig. 2 CT image shows healed enterocystoplasty.

to the colorectal team for completion and closure. A retropubic drain was also placed and the total operative time was 8 hours, in which bladder closure took 20 minutes.

One month postoperatively, cystography showed no contrast leakage around the patch (Fig. 3). Flexible cystoscopy showed the bovine pericardium patch in situ at the bladder dome, where it had integrated into the native tissue (Fig. 4). The catheter was removed and there was no urinary leakage or infection associated with the patch. Renal function was also preserved at baseline $(\mathrm{Cr} 83 \mathrm{mg} / \mathrm{dL}$, eGFR > 60). At the latest follow-up 15 months postoperatively, ultrasonography of the kidneys showed no hydronephrosis.

\section{DISCUSSION}

In this case report, both patients suffered from extensive loss of normal bladder walls and contractility, leading to thin-walled and poorly compliant bladders with small capacities. Common causes of this condition are: (a) previous irradiation to the pelvis secondary to radiotherapy for cancer; (b) previous bladder cancer with intravesical treatment leading to extensive inflammation; and (c) fibrosis and chronic inflammatory diseases of the bladder. ${ }^{(2)}$ It would have been challenging to perform tension-free primary closures of these bladders after resection because of the small bladder capacities and unhealthy tissue.

In such situations, the surgeon can choose to use bowel segments as a bladder wall substitute, as was done in Case 1. Though part of the patient's bowels were irradiated and had extensive adhesions, we were still able to obtain a short, healthy segment of the bowel for enterocystoplasty. As shown in Fig. 5, a short distal segment of the terminal small bowel (approximately $5 \mathrm{~cm}$ in length) with attached mesentery was used to close the bladder defect. The bowel segment was detubularised and stitched to the bladder wall without tension. Ideally, augmentation clam cystoplasty (Fig. 5), performed using a longer segment of bowel to decrease postoperative bladder pressure and enhance bladder compliance, would have been a better treatment choice. However, options were severely limited due to the small bladder capacity (approximately $50 \mathrm{cc}$ ) and extensive adhesions owing to multiple pelvic surgeries and previous pelvic irradiation. Performing augmentation clam cystoplasty would have been technically 


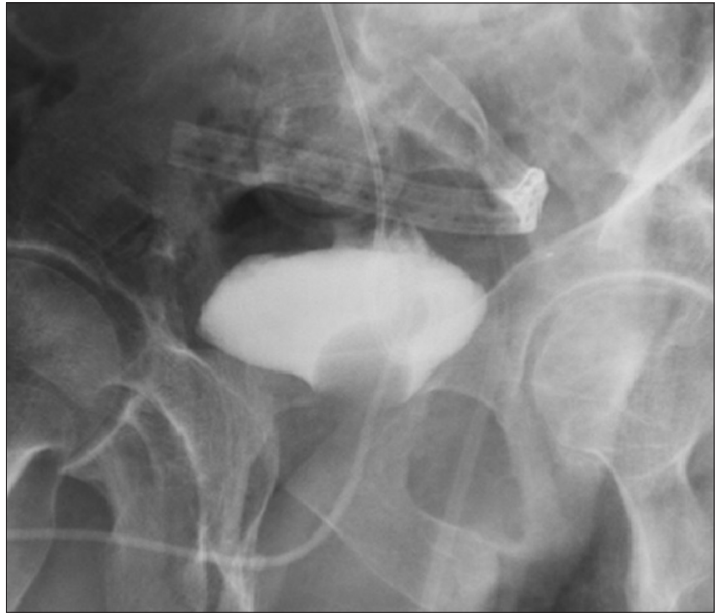

Fig. 3 Cystogram shows healed anastomosis of the bovine pericardial graft.

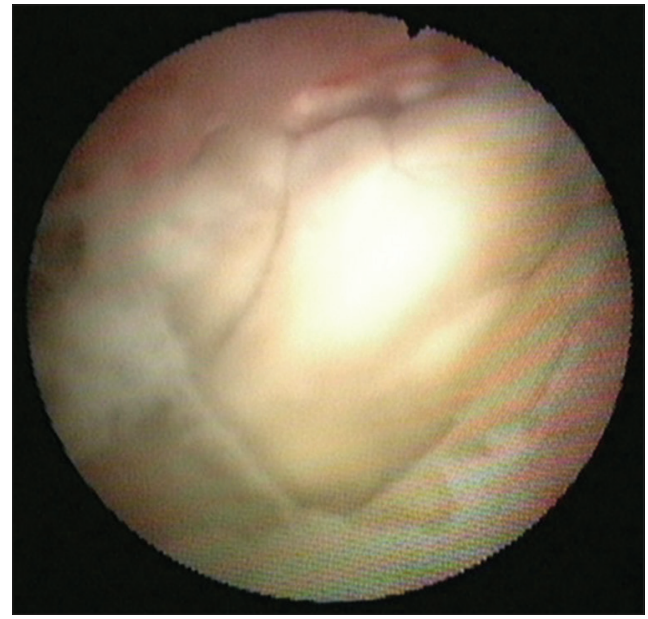

Fig. 4 Flex cystoscope shows the bovine pericardial graft four weeks after surgery.

challenging and increased the risk of further complications. More aggressive mobilisation of the already thin-walled bladder, which was extremely adherent to the surrounding structures, would have risked further inadvertent cystotomies. We were also worried about the healing capacity of the irradiated bladder and possibly irradiated small bowel. Additionally, we considered the possibility of a supravesical urinary diversion with ileal conduit. However, extensive and dense adhesions would render the dissection of the bilateral ureters difficult. We were also concerned about the use of the ileum as a conduit due to the patient undergoing concomitant laparotomy for intestinal obstruction. We thus opted for the use of a short segment of the ileum for bladder defect repair and augmentation of the bladder capacity. Postoperatively, there was a small anastomotic leakage, but it resolved with conservative management of bladder drainage. There was also an increased bladder capacity (approximately 80-100 cc). With a limited amount of healthy small bowel, an iatrogenic cystotomy can be reasonably repaired using an ileal patch, with acceptable complications. The use of the small bowel may facilitate in augmenting the small bladder capacity. Indeed, our first case addresses the issue of intraoperative closure and illustrates options for bladder defect repair in a desperate and complicated situation.
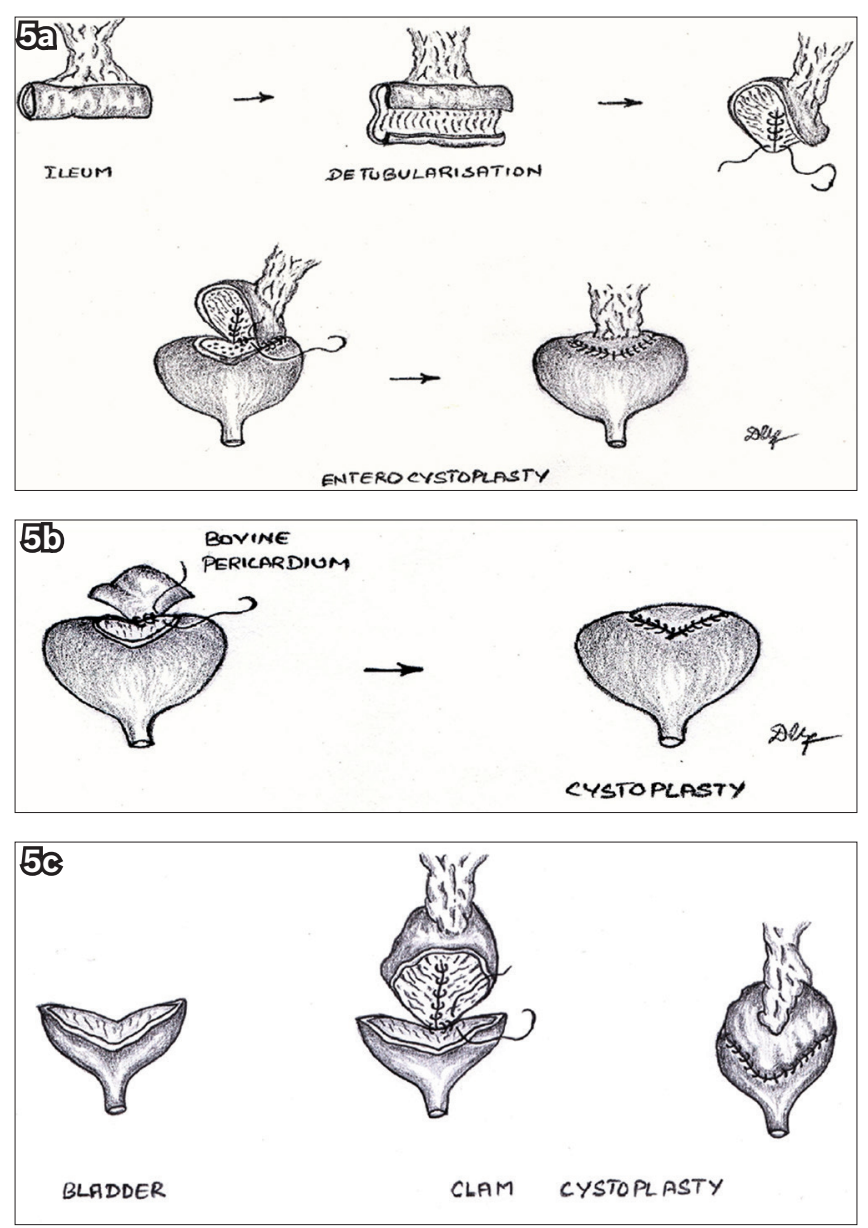

Fig. 5 Illustrations show (a) enterocystoplasty performed for Case 1; (b) bovine patch cystoplasty performed for Case 2; and (c) traditional clam cystoplasty.

In Case 2, bovine pericardium was chosen as a bladder wall substitute. The initial bladder capacity was small due to previous partial cystectomy and extensive pelvic irradiation. Rapid closure of the bladder defect was desirable due to an already prolonged and complicated operation. However, the bowel was unable to be used as a bladder repair material; it was extremely unhealthy due to previous irradiation and also densely adherent to the surrounding structures as a result of multiple previous laparatomies. The surgery went well and postoperative cystography did not reveal any leakage. The bovine pericardium patch managed to integrate well into the native tissue and the anastomosis healed well.

Bovine pericardium, though not widely used in urology, has been extensively used in other fields. It has been used to replace other kinds of tissue, such as cardiac valves, vessels, dura mater and tunica albuginea, with good results. ${ }^{(3)}$ The use of bovine pericardium has acceptable outcomes in vascular surgeries. ${ }^{(4)}$ In fact, the use of bovine pericardium is common and well established in vascular surgery, typically in patch angioplasty. ${ }^{(4)}$

The use of bovine pericardium as a bladder repair material has apparent benefits. Though it is a xenograft, it is biocompatible and has been proven to be non-immunogenic. ${ }^{(5)}$ In a randomised controlled study by Laun et al, ${ }^{(5)}$ comparison of the patients' 
Table I. Comparison of ileum and bovine pericardium.

\begin{tabular}{|c|c|c|}
\hline & Ileum & Bovine pericardium \\
\hline Advantages & $\begin{array}{l}\text { - Familiar graft to urologists } \\
\text { - Readily available } \\
\text { - Comes at no monetary cost }\end{array}$ & $\begin{array}{l}\text { - High strength }{ }^{(13)} \text { and durability } \\
\text { - Flexible }{ }^{(6,8)} \\
\text { - Low infection rate } \\
\text { (reported in other abdominal and urologic surgeries) }{ }^{(4)} \\
\text { - Easy to use and rapid repair } \\
\text { (i.e. shorter operative time, as shown in our cases) } \\
\text { - No bowel anastomosis required (i.e. no bowel leakage risk) }\end{array}$ \\
\hline Disadvantages & $\begin{array}{l}\text { - Development of metabolic acidosis } \\
\text { - Recurrence of urinary tract infections } \\
\text { - Potential calculi formation } \\
\text { - Risk of cancer (extrapolated from augmentation } \\
\text { cystoplasty series) }{ }^{(14)} \\
\text { - Small bowel may not be available if pelvis was irradiated }\end{array}$ & $\begin{array}{l}\text { - Relatively more expensive } \\
\text { - Potential calcification and calculi formation } \\
\text { - Rejection by surrounding tissue } \\
\text { - Adhesion to surrounding structures } \\
\text { - Allergic reaction }\end{array}$ \\
\hline
\end{tabular}

immunogenic response between the use of lyophilised bovine pericardium patch and lyophilised human dura mater patch for dura closure was made. It was concluded that bovine pericardium made for a better patch alternative, as no intensified immunogenic response was mounted. ${ }^{(5)}$ We utilised the bovine pericardium patch (Edwards Lifesciences, Irvine, CA, USA), which was fixed in glutaraldehyde for increased tissue stability and reduced antigenicity. ${ }^{(6)}$ The use of glutaraldehyde-fixed bovine pericardium patch has been shown to reduce antigenicity and hence graft rejection rates. ${ }^{(6)}$ Potential infections are also reduced. ${ }^{(4,7)}$ Bovine pericardium, being a fixed tissue, is cheap and readily available off the shelf. ${ }^{(4)}$ It also has the advantages of a high tensile strength and flexibility. It is elastic, as it contains a substantial amount of layered structural protein, and extremely durable. ${ }^{(6,8)}$ Fixation of bovine pericardium in glutaraldehyde also adds to its strength and stability. ${ }^{(4)}$ Well-known concerns about bovine pericardium expressed in other studies include that of calcification. ${ }^{(7)}$ However, the graft that we used in Case 2 makes use of a special anticalcification technology that reduces the incidence of calcification in the graft. ${ }^{(6,9)}$

Though the current use of bovine pericardium in the urological field is limited, few reports have highlighted its effectiveness as a bladder repair material. Novick et al were the first to conduct a canine experiment using bovine pericardium as a substitute for the upper half of the bladder wall. ${ }^{(10)}$ After 2.5 years, the authors found that there was replacement of the bovine pericardial tissue with epithelial tissue over the luminal surface, but no detrusor regeneration. ${ }^{(10)}$ Moon et al reported the first clinical case of bovine pericardium graft use in a patient with enterovesical fistula, after ileal cystoplasty was made impossible due to severe adhesions of the previously irradiated bowel. ${ }^{(11)}$ Our case (Case 2), which was the second clinical experience, showed that bovine pericardium graft can be used for such purpose.

In Table I, we compared the reported relative advantages and disadvantages of using an ileal and a bovine pericardial patch. ${ }^{(12)}$ When faced with an iatrogenic cystotomy in a small capacity bladder, the native irradiated bowel, which is better researched and widely used, can be used as a bladder wall substitute. However, if this is not possible, bovine pericardium may be a useful alternative. Although it is not widely used, its application in urology is promising and we anticipate greater usage of bovine pericardium by the urological community.

\section{REFERENCES}

1. Elbahnasy AM, Shalhav A, Hoenig DM, Figenshau R, Clayman RV. Bladder wall substitution with synthetic and non-intestinal organic materials. J Urol 1998; 159:628-37.

2. Rao PK, Iverson AJ, Sabanegh ES Jr. Augmentation cystoplasty. In: Medscape reference [online]. Available at: http://emedicine.medscape. com/article/443916-overview. Accessed May 10, 2013.

3. David TE. The use of pericardium in acquired heart disease: a review article. J Heart Valve Dis 1998; 7:13-8

4. Li X, Guo Y, Ziegler KR, et al. Current usage and future directions for the bovine pericardial patch. Ann Vasc Surg 2011; 25:561-8.

5. Laun A, Tonn JC, Jerusalem C. Comparative study of lyophilized human dura mater and lyophilized bovine pericardium as dural substitutes in neurosurgery. Acta Neurochir (Wien) 1990; 107:16-21.

6. Edwards Bovine Pericardial Patch. In: Edwards Lifesciences [online] Available at: http://www.edwards.com/products/porcinevalves/pages/ patch.aspx. Accessed October 21, 2013.

7. García Páez JM, Jorge-Herrero E. Assessment of pericardium in cardiac bioprotheses. A review. J Biomater Appl 1999; 13:351-88.

8. Vesely I, Mako WJ. Comparison of the compressive buckling of porcine aortic valve cusps and bovine pericardium. J Heart Valve Dis 1998; 7:34-9.

9. Cunanan CM, Cabiling CM, Dinh TT, et al. Tissue characterization and calcification potential of commercial bioprosthetic heart valves. Ann Thorac Surg 2001; 71(5 Suppl):S417-21.

10. Novick AC, Straffon RA, Koshino I, et al. Experimental bladder substitution using a biodegradable graft of natural tissue. J Biomed Mater Res 1978; 12:125-47.

11. Moon SJ, Kim DH, Jo JK, et al. Bladder reconstruction using bovine pericardium in a case of enterovesical fistula. Korean J Urol 2011; 52:150-3.

12. Portis AJ, Elbahnasy AM, Shalhav AL, et al. Laparoscopic augmentation cystoplasty with different biodegradable grafts in an animal model. J Urol 2000; 164:1405-11.

13. Bellows CF, Alder A, Helton WS. Abdominal wall reconstruction using biological tissue grafts: present status and future opportunities. Expert Rev Med Devices 2006; 3:657-75.

14. Venn S, Mundy T. Bladder reconstruction: urothelial augmentation, trauma, fistula. Curr Opin Urol 2002; 12:201-3. 